\title{
Complete genome of Staphylococcus aureus Tager 104 provides evidence of its relation to modern systemic hospital-acquired strains
}

\author{
Richard W. Davis IV', Andrew D. Brannen', Mohammad J. Hossain², Scott Monsma³, Paul E. Bock4, \\ Matthias Nahrendorf ${ }^{5}$, David Mead ${ }^{3}$, Michael Lodes ${ }^{3}$, Mark R. Liles $^{2}$ and Peter Panizzi ${ }^{1 *}$ (D)
}

\begin{abstract}
Background: Staphylococcus aureus (S. aureus) infections range in severity due to expression of certain virulence factors encoded on mobile genetic elements (MGE). As such, characterization of these MGE, as well as single nucleotide polymorphisms, is of high clinical and microbiological importance. To understand the evolution of these dangerous pathogens, it is paramount to define reference strains that may predate MGE acquisition. One such candidate is S. aureus Tager 104, a previously uncharacterized strain isolated from a patient with impetigo in 1947.

Results: We show here that $S$. aureus Tager 104 can survive in the bloodstream and infect naïve organs. We also demonstrate a procedure to construct and validate the assembly of S. aureus genomes, using Tager 104 as a proofof-concept. In so doing, we bridged confounding gap regions that limited our initial attempts to close this $2.82 \mathrm{Mb}$ genome, through integration of data from Illumina Nextera paired-end, PacBio RS, and Lucigen NxSeq mate-pair libraries. Furthermore, we provide independent confirmation of our segmental arrangement of the Tager 104 genome by the sole use of Lucigen NxSeq libraries filled by paired-end MiSeq reads and alignment with SPAdes software. Genomic analysis of Tager 104 revealed limited MGE, and a vSa $\beta$ island configuration that is reminiscent of other hospital acquired S. aureus genomes.
\end{abstract}

Conclusions: Tager 104 represents an early-branching ancestor of certain hospital-acquired strains. Combined with its earlier isolation date and limited content of MGE, Tager 104 can serve as a viable reference for future comparative genome studies.

\section{Background}

The prevalence of methicillin-resistance in Staphylococcus aureus (S. aureus) is a global threat as noted by the Centers for Disease Control and Prevention 2013 Threat Report [1]. S. aureus strains are heterogeneous, and therefore can cause a wide range of localized infections, such as impetigo and cellulitis, to more serious systemic infections, such as bacterial endocarditis and sepsis. In particular, S. aureus is the leading cause of bacterial endocarditis, a disease with mortality rates as high as $25-47 \%$, even in the presence of antibiotic therapy [2]. This wide range of

\footnotetext{
*Correspondence: panizzi@auburn.edu

1 Department of Drug Discovery and Development, Harrison School of Pharmacy, Auburn University, 4306 Walker Building, Auburn, AL 36849, USA Full list of author information is available at the end of the article
}

infections, as well as the growing number of pathogens with diverse antibiotic resistance profiles, is due to the acquisition of mobile genetic elements (MGEs) that grant heterogeneity to $S$. aureus strains. The best described of these MGEs is the Staphylococcal Cassette Chromosome mec (SCCmec) operon, which encodes the mecA gene and confers methicillin resistance [3]. However, many other MGEs can confer adaptive advantages during infection, such as enterotoxins [4], leukocidins (most prominently the Panton-Valentine leukocidin, $p v l$, encoded by the $l u k F$ $P V$ and $l u k S-P V$ genes) [3, 4], staphylokinase (sak) [5], and the toxic shock syndrome toxin-1 [6]. Therefore, there is an urgent need to better understand the evolutionary adaptations commonly associated with acquisition of MGE that contribute to (i) the prevalence of nosocomial infections, 
(ii) the rapid spread of community-acquired strains, (iii) development and expansion of novel resistance mechanisms, and (iv) natural host selection and propagation pathways.

To juxtapose recent next-generation sequencing efforts with pathogenic potential, it is necessary to have several well-characterized reference strains to use as landmarks to interpret MGE findings. This is currently lacking in the field, as the most popular strains (i.e. methicillin-sensitive S. aureus (MSSA) Newman [7], methicillin-resistant $S$. aureus (MRSA) USA300 [8], and vancomycin-resistant $S$. aureus (VRSA) Mu50 [4, 9]) are complicated by horizontal gene transfer of MGE and inclusion of various prophages that were gained to most likely provide some sort of survival advantage. As such, it is prudent to define strains with a balance between limited MGE presence and potent pathogenic potential.

Here we describe one such strain, which we term $S$. aureus Tager 104. Tager 104 was originally isolated at the New Haven Hospital (New Haven, Connecticut) by Morris Tager et al. in 1947 from a patient with a cutaneous infection caused by a hemolytic bacteria $[10,11]$. As a comparison, the first strain of methicillin-resistant $S$. aureus was isolated in 1961 [3]. Morris Tager and coworkers subsequently demonstrated that $S$. aureus Tager 104 induced clotting through expression of secreted factors [12], developed staphylocoagulase purification protocols [13], and initiated preliminary characterization of staphylocoagulase function in contrast to normal physiologic clotting $[14,15]$. Currently, staphylocoagulase from S. aureus Tager 104 is arguably the most well characterized prothrombin activator studied to date, as it was the original source used to solve the staphylocoagulase (1-325) fragment crystal structure in complex with both thrombin and its immediate precursor, prethrombin 2 [16]. This recombinant $\mathrm{N}$-terminal fragment of staphylocoagulase from Tager 104 binds with high affinity $\left(K_{\mathrm{D}} 17-72 \mathrm{pM}\right)$ to the human prothrombin zymogen [17], which was also used to characterize fibrinogen recognition by the prothrombin-staphylocoagulase complex and to determine that two prothrombin-staphylocoagulase complexes bind to a single substrate fibrinogen molecule to mediate cleavage [18]. With the wealth of biochemical insight that has been generated from Tager 104 virulence factors, the genomic characterization has merit from both clinical and evolutionary perspectives.

\section{Methods}

\section{Ethics statement}

For this study, C57BL/6 mice were purchased from The Jackson Laboratories (Bar Harbor, ME). Mice were housed at Auburn University College of Veterinary Medicine with ad libitum access to alfalfa-free chow and water. All procedures were designed in accordance with the Guide for the Care and Use of Laboratory Animals of the National Institutes of Health and approved by the Institutional Animal Care and Use Committee of Auburn University for this sepsis model under protocol 2014-2427. S. aureus strain Tager 104 was obtained as a depersonalized human isolate from an outside source and therefore no ethics approval was required for its use in this study.

\section{Mouse model of systemic infection}

For this experiment, 12 female C57BL/6 were anesthetized with 1-3 \% isoflurane mixed with medical grade oxygen using a vaporizer. S. aureus Tager 104 was grown overnight at $37{ }^{\circ} \mathrm{C}$ in $50 \mathrm{~mL}$ of brain-heart infusion (BHI) broth in a $125 \mathrm{~mL}$ Erlenmeyer flask. Cells were twice washed with $40 \mathrm{~mL}$ of filter-sterilized phosphate buffered saline (PBS) supplemented with $10 \%$ glycerol. Cells were pelleted by centrifugation at $10,000 \times g$ for $10 \mathrm{~min}$ and the cell pellet was re-suspended in a final volume of $10 \mathrm{~mL}$ of PBS with $10 \%$ glycerol. A sample from this inoculum stock was diluted 1:100 in PBS with $10 \%$ glycerol in a cuvette for determination of cell density by measuring the absorbance at an optical density (O.D.) at $600 \mathrm{~nm}$ using a Beckman Coulter DU800 spectrophotometer. These freshly prepared inoculums were injected into these 12 female C57BL/6 mice by tail-vein at a dose of $5 \times 10^{8}$ colony forming units (CFU). After $24 \mathrm{~h}$, the animals were euthanized.

\section{Histology}

Organs were harvested, stored in $4 \%$ paraformaldehyde, and embedded in optimal cutting temperature (OCT) medium by immersing in 4-methylbutane in a metal canister within a dry-ice bath. Slices $(10$ microns in width) were made using a Thermo HM550 Cryostat set at $-19^{\circ} \mathrm{C}$, and fixed to poly-L-lysine coated slides. Slides were then stained using standard Gram and hematoxylin and eosin $(\mathrm{H} \& \mathrm{E})$ staining protocols. Slides were viewed at 100 or 400 times total magnification on a Zeiss Axioskop 40 microscope, and images were taken using a Nikon DS-Fi1 camera head and DS-L3 control unit.

\section{Lucigen NxSeq library construction}

Genomic DNAs were prepared by E.Z.N.A. Bacterial D.N.A. kit (Omega Bio-tek, Norcross, GA). All genomic DNA preparations were evaluated for approximate size and integrity by Pulsed Field Gel Electrophoresis with a BioRad CHEF-DR III instrument. Fragment Libraries were constructed with a NxSeq DNA Sample Prep Kit (Lucigen). Briefly, genomic DNAs were sheared to approximately 500 to $700 \mathrm{bp}$ with a Covaris instrument and fragment size was confirmed by agarose gel electrophoresis. Five hundred ng sheared DNA was mixed with $2 \mathrm{X}$ buffer and a cocktail of end repair and tailing 
enzymes and then incubated at $25^{\circ} \mathrm{C}$ for $20 \mathrm{~min}$ and then $72{ }^{\circ} \mathrm{C}$ for $25 \mathrm{~min}$. Illumina sequencing adaptors and ligase were then added and the tubes were incubated at $25{ }^{\circ} \mathrm{C}$ for 30 min prior to size selection with Agencourt AMPure XP beads (Beckman Coulter). Fragment libraries were then quantified with a 2100 Bioanalyzer (Agilent Technologies), sequenced on a MiSeq Sequencer (Illumina) and $2 \times 250$ paired end reads were exported for analysis.

\section{Mate pair libraries}

Insert preparation, size selection and library construction followed the $\mathrm{NxSeq}^{\circ} 8 \mathrm{~kb}$ Long Mate Pair Library Kit. Briefly, $8 \mathrm{~kb}$ mate pair library insert was sheared to approximately $10 \mathrm{~kb}$ with either a Megaruptor (Diagenode Inc., Denville, NJ) or a g-TUBE (Covaris, Woburn, MA). Sheared DNA was bead-cleaned and then size-selected with Agencourt AMPure XP beads. After size selection and quantification with a Qubit 2.0 Fluorometer (ThermoFisher), $400 \mathrm{ng}$ of insert was ligated to a coupler at a concentration of $1 \mathrm{ng} / \mu \mathrm{L}$ for $16 \mathrm{~h}$ at $16{ }^{\circ} \mathrm{C}$. Unligated DNA was removed by digestion with exonucleases and the circularized DNA was purified with Agencourt AMPure XP beads. Purified DNA was then digested with endonucleases (ThermoFisher) to remove DNA between the two ditags, and the ditag-ligated coupler was purified by biotin capture with MyOne $\mathrm{C} 1$ streptavidin magnetic beads (ThermoFisher). The purified ditag-ligated coupler was then G-tailed and ligated to a C-Tailed Junction Code adaptor. After bead cleaning to remove unligated adaptor, the Junction Code adapted DNA was re-circularized at low concentration, cleaned with Agencourt AMPure XP beads and then amplified with Accura HF Hot Start Master Mix (Lucigen) and indexed primers.

Construction of $20 \mathrm{~kb}$ mate pair libraries followed the $\mathrm{NxSeq}^{\circ} 20$ kb Long Mate Pair Library Kit protocol. Briefly, $15 \mu \mathrm{g}$ of high molecular weight genomic DNA was sheared to $20 \mathrm{~kb}$ with a g-TUBE at $4500 \mathrm{rpm}$, end repaired, A-tailed and ligated to adaptors prior to precipitation and resuspension in $10 \mathrm{mM}$ Tris. Adapted insert was gel-isolated either with a BluePippin instrument (Sage Science) or by separation on a $0.3 \%$ SeaKem Gold agarose (Lonza) followed by electroelution with an EluTrap device (Whatman). Gel-isolated insert was then quantified by Qubit and $1 \mu \mathrm{g}$ of insert was ligated to a coupler at a concentration of $1.25 \mathrm{ng} / \mu \mathrm{L}$ for $16 \mathrm{~h}$ at $4{ }^{\circ} \mathrm{C}$. Unligated DNA was removed by digestion with exonucleases and the circularized DNA was purified by alcohol precipitation. The $20 \mathrm{~kb}$ protocol continues with endonuclease digestion as described above for the $8 \mathrm{~kb}$ mate pair protocol. Mate pair libraries were quantified with a 2100 Bioanalyzer, sequenced on a MiSeq Sequencer (Illumina) and $2 \times 250$ paired end reads were exported for subsequent filtering and trimming.
True mate pairs were filtered by analysis and trimming of Chimera Code coupler sequences using the Chimera-Clean5 Python script (Lucigen); junctions between left and right di-tags were identified by presence of the Junction Code linker and di-tags extracted using the JunctionSplit9 Python script (Lucigen).

\section{Tager 104 genome construction}

Genomic DNA was extracted from Tager 104 using E.Z.N.A. Bacterial D.N.A. kit (Omega Bio-tek, Norcross, GA) and constructed into a bar-coded library using the Nextera DNA sample preparation kit (Illumina, San Diego, CA). Sequencing was performed using an Illumina MiSeq sequencer for $2 \times 150$ paired end reads and trimmed sequence reads were assembled de novo using CLC Bio v. 4.6.1, as described previously [19]. To scaffold these contigs, a sub-library of Tager 104 was constructed for PacBio SMRT sequencing. Two sequencing reactions were performed, and CLC bio contigs were scaffolded using Celera Assembler pipeline on the SMRT analysis 1.3 suite [19].

To overcome the innate difficulty in genome closure in this initial construction, PacBio reads were instead assembled de novo using SMRT Analysis v. 2.0 Hierarchical Genome Assembly Process (HGAP) algorithm [20], which produced 8 contigs. To close the genome, two Lucigen NxSeq $20 \mathrm{~kb}$ mate-pair libraries were constructed and sequenced on an Illumina HiSeq system. PacBio HGAP scaffolds and Lucigen $\mathrm{NxSeq}$ paired-end reads from the mate-pair library were provided to SSAKE-based scaffolding of Pre-Assembled Contigs after Extension (SSPACE) [21] for de novo assembly to create the closed draft genome. Gap regions in this genome were closed using a combination of the GapFiller algorithm (as part of the SSPACE suite) and Basic Local Alignment Search Tool (BLAST) search against the initial CLC contigs for those which bridge gap regions. This complete, circular genome was submitted to the Rapid Annotation using Subsystem Technology (RAST) server [22-24].

To confirm the construction of Tager 104 using an independent method, new libraries were constructed using the Nextera DNA kit and sequenced using Illumina MiSeq $2 \times 250$ reactions. These results were combined using the St. Petersburg genome assembler (SPAdes) algorithm [25] for genome closure.

\section{Construction validation and analysis}

To test the contribution of repeats to the shortcomings in genome assembly, long repeats ( $>500 \mathrm{bp}$ ) were identified using Nucmer mapping of the Tager 104 genome to itself [26] and selecting for regions with unique locations and proper size. In addition, interspersed repeats and 
RNA sequences were identified using the RepeatMasker algorithm (www.repeatmasker.org). The coordinates of unique repeats were recorded and provided to Circos version 0.64 (www.circos.ca).

To determine the contribution of Illumina MiSeq contigs (constructed using CLC), PacBio RS sequencing reads, and scaffolds constructed from the combination of the two, results from each assembly were mapped to the Tager 104 genome using Nucmer. The locations of each unique mapping were provided to Circos for visualization.

\section{MLST analysis}

To compare the lineage of Tager 104 to other clinical $S$. aureus strains, we employed multi-locus sequence typing (MLST). FASTA-formatted genomic sequences of completed $S$. aureus genomes were obtained from the RefSeq database on GenBank and uploaded to CLC Workbench. The identity of the seven housekeeping genes (carbamate kinase, arc; shikimate dehydrogenase, aro; glycerol kinase, $g l p$; guanylate kinase, gmk; phosphate acetyltransferase, $p t a$; triosephosphate isomerase, tpi; acetyl coenzyme A acetyltransferase, $y q i$ ) were determined in silico using the MLST module (http://www.clcbio.com/clc-plugin/mlst/). The sequence type of Tager 104 was determined previously to be ST49, as outlined elsewhere [19].

\section{Proteomic analysis of Tager 104 genome}

To perform whole-genome comparisons of Tager 104 to reference $S$. aureus strains, a CMG-Biotools virtual machine was graciously provided by Dr. David Ussery [27]. One reference genome was selected from each ST group represented in MLST analysis. Each genome sequence was downloaded from GenBank using the getgbk (i.e., content) function, and converted into FASTA files using saco_convert. These files were then submitted to the prodigalrunner algorithm to process the open reading frames and create a protein FASTA file. The makebmdest and blastmatrix algorithms were then utilized to construct BLAST matrices using one representative from each ST.

\section{Whole-genome phylogenetic analysis}

The Tager 104 genome and the GenBank reference sequence genomes were obtained from RAST and GenBank, respectively. Genomes were submitted to alignment by the Mauve algorithm using default parameters [28]. Orthologs were extracted from the resulting .xmfa file using stripSubsetLCBs (http://darlinglab.org/mauve/snapshots/2015/2015-01-09/linux-x64/) with the minimum locally colinear blocks length set to $500 \mathrm{bp}$ and the number of genomes set to 75 . The result was then converted to FASTA format using the xmfa2fasta.pl script, and the FASTA-formatted alignment was then converted to phylip format using the fasta2phylip.pl script (both parts of the BioPerl package) (www.bioperl.org). To construct the phylogenetic tree, a maximum-likelihood tree was constructed using the RAxML program on the Cyberinfrastructure for Phylogenetic Research (CIPRES) Science Gateway using default settings (www.phylo.org/portal2/ home.action), and the image was constructed using FigTree (tree.bio.ed.ac.uk/software/figtree).

\section{MGE-encoded gene determinations}

The list of MGE-encoded predicted virulence factors was determined based on previous studies by our lab and others, and reviewed elsewhere [3]. The RAST server was first searched for the presence of each proteinencoding gene (PEG). In cases where no annotated PEG was detected, the UniProt server (www.uniprot.org) was used to obtain DNA sequences for BLAST analysis. The genetic location of these elements was recorded and provided to Circos for graphic representation.

To compare genomes of the Tager clade, we utilized the BLAST Ring Image Generator (BRIG) algorithm (http://brig.sourceforge.net/) with default parameters [29].

Genomic islands were determined using the IslandViewer 3 algorithm, available online at http://www.patho genomics.sfu.ca/islandviewer. Islands were identified based on their genetic content, as reported by the IslandViewer output and BLAST analysis.

For the $v \mathrm{Sa} \beta$ genomic island, genomic profiles were constructed using the RAST server. After determination of the profile, open reading frames were recolored based on their family or function.

\section{Antibiotic susceptibility testing}

S. aureus Tager 104 was streaked for a lawn on trypticase soy agar (TSA) plates containing $5 \%$ sheep blood (BD Biosciences). E-test strips for erythromycin (ER), linezolid (LZ), oxacillin (OX), and vancomycin (VA) (bioMérieux, Inc.) were then placed onto the surface of the agar using sterile forceps. All resistance tests were performed under conditions that were naïve for the $S$. aureus Tager 104 strain to not complicate interpretation by inducing vancomycin-resistance. Bacteria were incubated at $37{ }^{\circ} \mathrm{C}$ for $17 \mathrm{~h}$ and plates were imaged using an IVIS Lumina XR system. In addition, overnight cultures of $S$. aureus Tager 104 were added at a 1:200 ratio to wells containing BHI broth and serial diluted concentrations of vancomycin. The 96-well plate was incubated for $17 \mathrm{~h}$ in an incubator and the O.D.600nm was determined using a Thermo Scientific VarioSkan plate reader. Data was subsequently plotted using Graph Pad Prism v4.03.

\section{Results}

S. aureus Tager 104 abscess formation in naïve tissue

Histology indicates that $S$. aureus Tager 104 is able to colonize all tissue with variable coverage at this time 
point as indicated by Gram staining (Fig. 1). Bacteria were detected in the alveoli cells of the lung (not shown), the white pulp of the spleen, and glomerulus of the kidney. This supports the previous hypothesis that Tager 104 is capable of systemic spread. Given our previous studies [30, 31], we now know that Tager 104 can cause both endocarditis and establish abscesses in naive tissue. We sought to better characterize this Tager 104 at a genomic level to consider all potential virulence determinants prior to comparative virulence studies with other S. aureus strains.

\section{Construction of S. aureus Tager 104 genome}

To close the $S$. aureus Tager 104 genome, three different sequencing technologies were used: Illumina Miseq $2 \times$ 150 bp reactions, PacBio RS 90-min movies, and Lucigen $\mathrm{NxSeq} 20 \mathrm{~kb}$ mate-pair libraries. In all approaches, scaffolds were constructed using one of either Illumina MiSeq or PacBio RS, or a combination of the two (hybrid assembly).

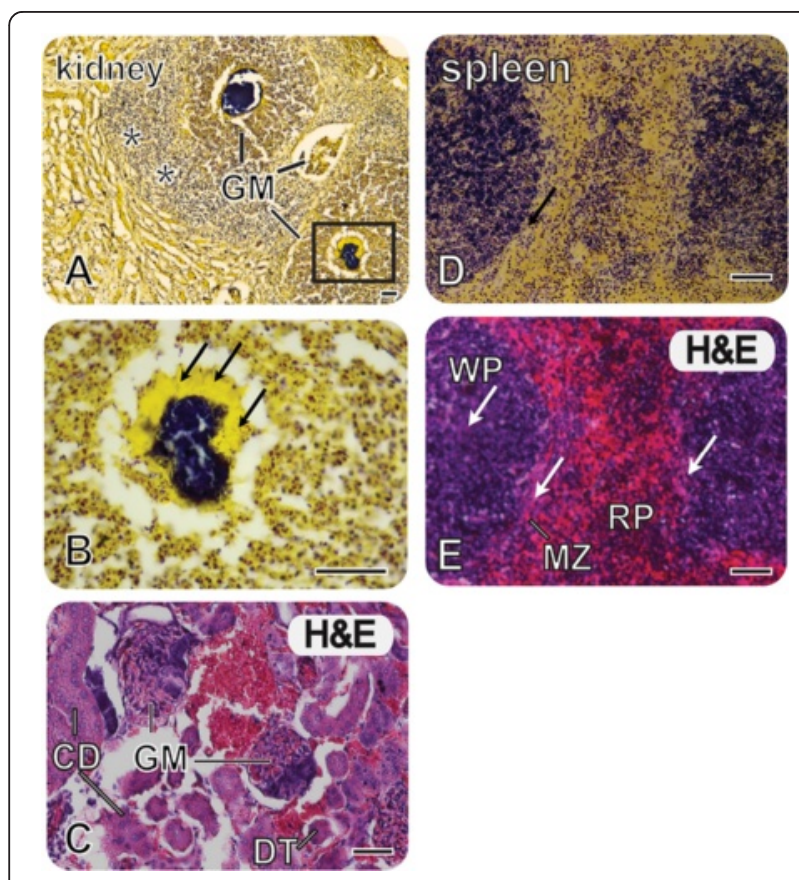

Fig. 1 S. aureus Tager 104 Bacteremia Leading to Multi-organ Septic Foci. a Gram staining of C57BL/6 kidney 8 days after injection of $5 \times 10^{8}$ CFU S. aureus Tager 104. b Higher magnification (400× total) of the indicated by the box in panel a. Arrows indicate the protective fibrin layer that allows the bacteria to thrive in the glomerulus (GM) without the threat of clearance by phagocytic immune cells (see area immediately surrounding infected GM shown in Panel a). c Adjacent section of the same kidney stained with Hematoxylin and Eosin (H\&E); please note the GM is at a slightly earlier stage of the disease. Kidney architecture including the collecting ducts (CD), the distal tubule (DT), and blood cells (red). d Gram staining shows bacteria (purple) in all organs examined; splenic abscesses also show thickening of the marginal zone and increased fibrin deposition (arrows). e Adjacent section stained for H\&E showing the splenic architecture including the white pulp (WP) region, marginal zone $(M Z)$ and red pulp $(R P)$ region
We first sought to close the hybrid assembly scaffolds previously produced for Tager 104 [19]. Therefore, these scaffolds were submitted to the SSPACE algorithm with the Lucigen NxSeq mate pair sequences. This approach successfully closed the genome, producing the $2.8 \mathrm{Mb} S$. aureus genome. However, this approach left six large gaps in the assembly, which would have required significant manual closure.

Therefore, we instead tested the ability of PacBio RS sequencing reactions in combination with NxSeq matepair libraries. Scaffolds were produced using the Hierarchical Genome Assembly Process (HGAP) assembly algorithm on the PacBio SMRT Analysis suite (v 2.0) [20]. This algorithm produced 8 scaffolds from raw sequencing data, with an N50 value of 1,028,373 bp. These scaffolds were then closed using de-duplicated $\mathrm{NxSeq}$ reads using SSPACE. When possible, small gaps were filled by aligning CLC-constructed Illumina contigs to the genome using a BLAST search. The result was a closed 2.82 Mbp genome (Fig. 2a).

To independently confirm our scaffolding of the putative $2.82 \mathrm{Mbp}$ genome, Illumina MiSeq $2 \times 250$ libraries were constructed and sequenced using Illumina $2 \times 250$ sequencing reactions, and the resulting contigs were scaffolded using Lucigen NxSeq reactions. The resulting closed genome was identical to that produced by the hybrid HGAP-SSPACE assembly pipeline (Fig. 2b).

\section{Repeat regions created difficulty in automated closure}

To determine the shortcomings of individual methods on the closing of the Tager 104 genome, as a model for future $S$. aureus genome assembly, each contig or scaffold was mapped to the final genome using Nucmer and visualized using Circos (Fig. 3). In addition, repeats were determined by mapping the Tager genome to itself and selecting for matches with unique locations and a size $>$ 500 bp. Interspersed repeats and RNA sequences were determined using the RepeatMasker algorithm. Based on the analysis, Illumina MiSeq $2 \times 150$ reads did not properly bridge areas of the genome that were rich in repeats. More specifically, repeats $>500$ bp (Fig. 3, Region 1) and RNA repeats (Fig. 3, Region 2) caused the greatest issues in construction, as visualized. In addition, PacBio RS coverage was low across these areas. Therefore, information provided by the Lucigen NxSeq sequencing reactions was essential for the proper untangling and assignment of these genomic regions.

\section{Proteomic analysis of $S$. aureus reference sequences}

Delineation of the $S$. aureus strains is typically achieved using MLST analysis. Briefly, the analysis utilizes sequence heterogeneity among seven housekeeping genes to provide a higher resolution phylogenetic analysis. 


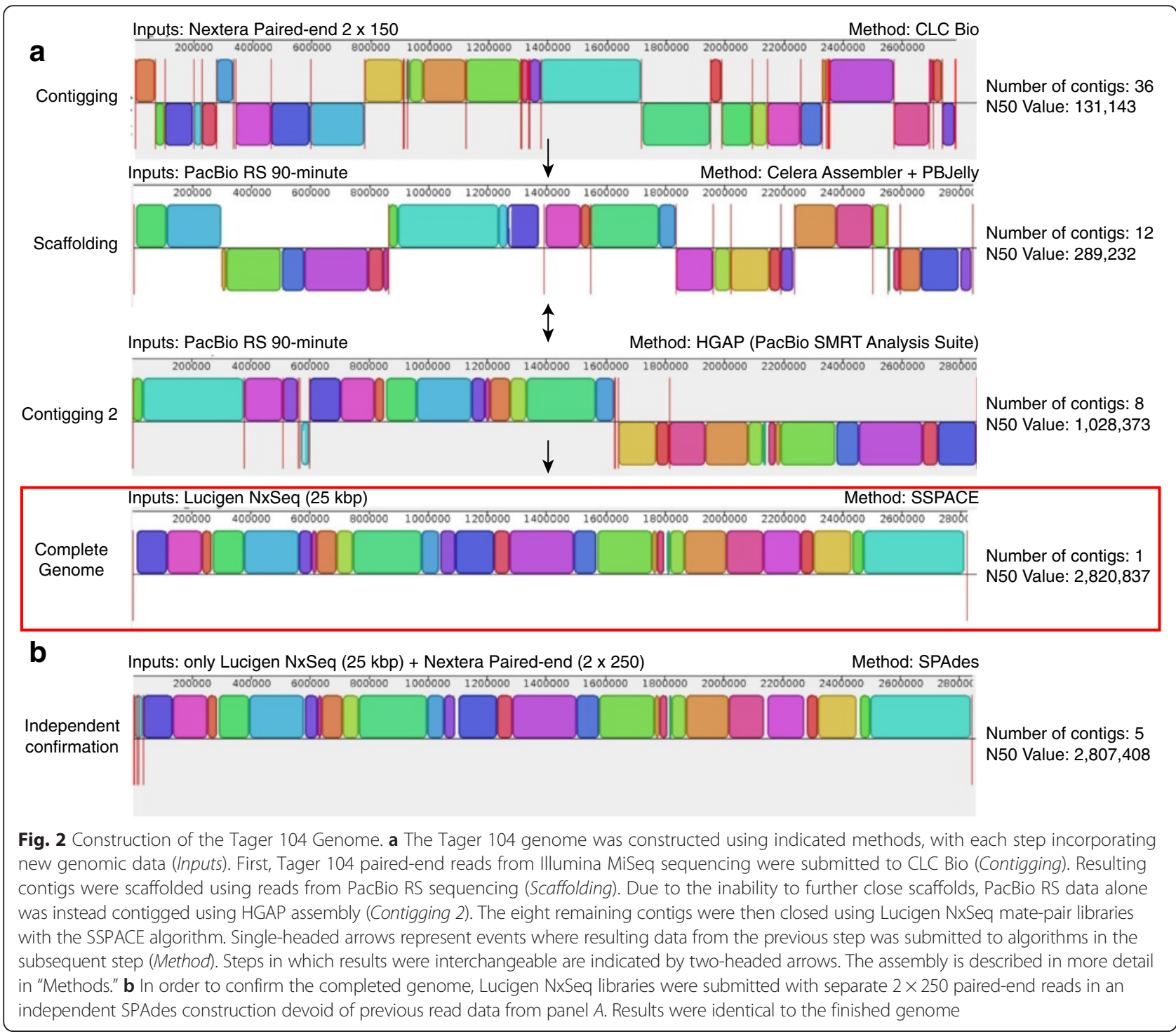

Reference genomes were obtained from the GenBank RefSeq database (Additional file 1: Table S1). MLST and spa typing of S. aureus Tager 104 previously revealed it to be ST49, the predicted founder of clonal complex 49 (CC49) [19]. CC49 was also shown to contain ST138, ST1693, ST1937, and ST2273 based on eBurst analysis (data not shown) [32].

To determine the similarities of protein content in completed sequences of $S$. aureus, each genome was called for open reading frames using the prodigalrunner algorithm and compared using the blastmatrix algorithm on CMG-Biotools version 2.2. The results shown in Fig. 4 indicate that the Tager 104 proteome is most closely related to Newman, a strain isolated in 1952 from a human patient and which has been well characterized for its coagulase activity. Similarity in these genomes supports the previously observed similarity in endocarditis models [30]. Following this strain, Tager 104 was $>84 \%$ similar to the nosocomial MRSA strain 04-02981, community-acquired MRSA strain SA268, and the human pleural isolate DSM 20231. The greatest proteomic differences were seen for strain ST398, a typically ovine-associated strain isolated from a human case of endocarditis, which was also the second-largest proteomic divergence observed [33].

\section{Tager 104 is an ancestor to clinical isolates}

To determine the lineage of $S$. aureus reference strains in relation to Tager 104, genomes were submitted to Mauve alignment and conserved segments were stripped and concatenated for each genome for phylogenetic analysis by RAxML. This alignment and phylogeny used only core genomic regions present in all $75 \mathrm{~S}$. aureus strains available as of Oct. $1^{\text {st }} 2015$.

The resulting tree (Fig. 5 and Additional file 2: Figure S1) shows Tager 104 to be the earliest branching member of a 


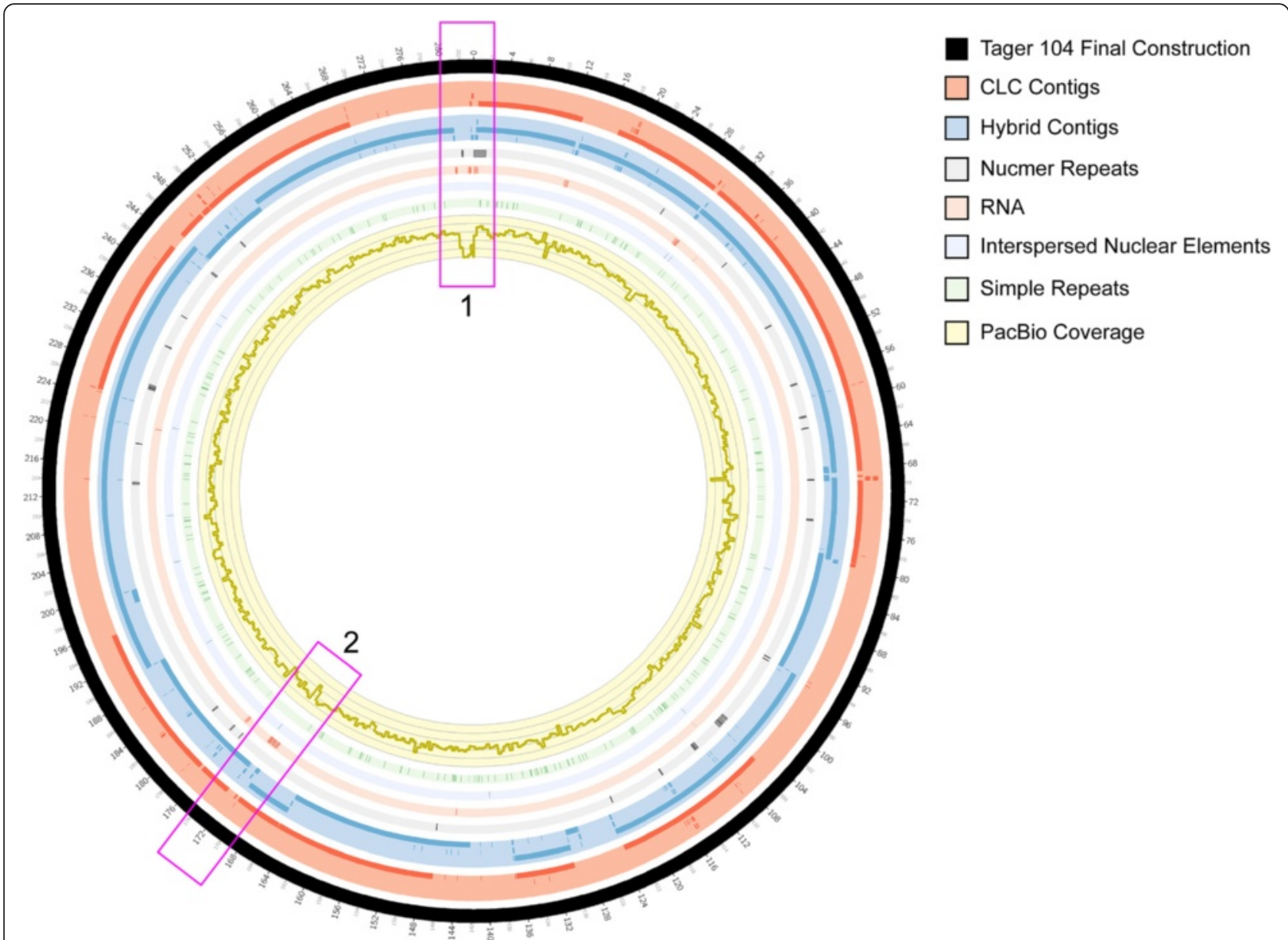

Fig. 3 Graphical Depiction of Tager 104 Assembly. The Tager 104 genome was scaffolded using three separate technologies: contigs from MiSeq reactions (red tiling), PacBio RS reads (yellow histogram), and scaffolds produced from the hybrid assembly of both (blue tiling). However, assembly of the Tager 104 genome using these technologies were found insufficient alone, due to the presence of repeat regions. More specifically, long repeats (>500 bp) create errors in de Bruijn graph untangling (gray tiling). In addition, the exact placement of short repeats, such as interspersed nuclear elements (light blue tiling), RNA sequences (light red tiling) and simple repeats (light green tiling) create errors in construction. Areas rich in repeats and low in sequencing coverage (Regions 1 and 2) reveal difficulties in genomic construction. These difficulties were overcome by providing the construction algorithms with 20 kbp mate-pair information, provided by Lucigen NxSeq libraries

clade containing strain 6850, M013, SA40, SA268, SA957, ED133, and RF122. Strain 6850 was isolated from a skin abscess at the University of Wisconsin in 1987, and was shown to progress from the abscess to complications such as systemic abscesses and septic arthritis [34]. This supports Tager 104 as a reference sequence for systemically-penetrating bacteria. As a positive control, 2 separate genome dataset corresponding to COL and NRS100 were also used and there is indication that these strains are the same or closely related. The results of our analysis are consistent with this hypothesis.

\section{Tager 104 contains a limited set of MGE-encoded} virulence factors targeted at host immunity

As Tager 104 was able to survive in septic murine models, and due to its phylogenetic placement as an ancestral S. aureus lineage, we investigated the genomic content of MGE-encoded virulence factors that have been previously identified and characterized [3]. The results demonstrate a limited set of encoded factors in the Tager 104 genome that are predicted to be necessary for bacterial survival during bacteremia (Fig. 6a). In addition, BRIG analysis revealed similar genomic regions between strains of the Tager clade (Fig. 6b).

Tager 104 is predicted to encode a $v$ Sa $\alpha$-encoded Staphylococcal super-antigen-like SSL, as well as $v S a \beta-$ encoded leukotoxin D and $\mathrm{E}$ ( $l u k D$ and $l u k E$, respectively). These factors are known to inhibit elements of innate immunity [35]. The $v \mathrm{Sa} \beta$-island was also shown to contain the hyaluronate lyase gene (hys $A$ ), which degrades hyaluronic acid found in host extracellular matrix.

The Tager 104 genome also indicates early exposure to phage-carried virulence factors. Tager 104 contained the 


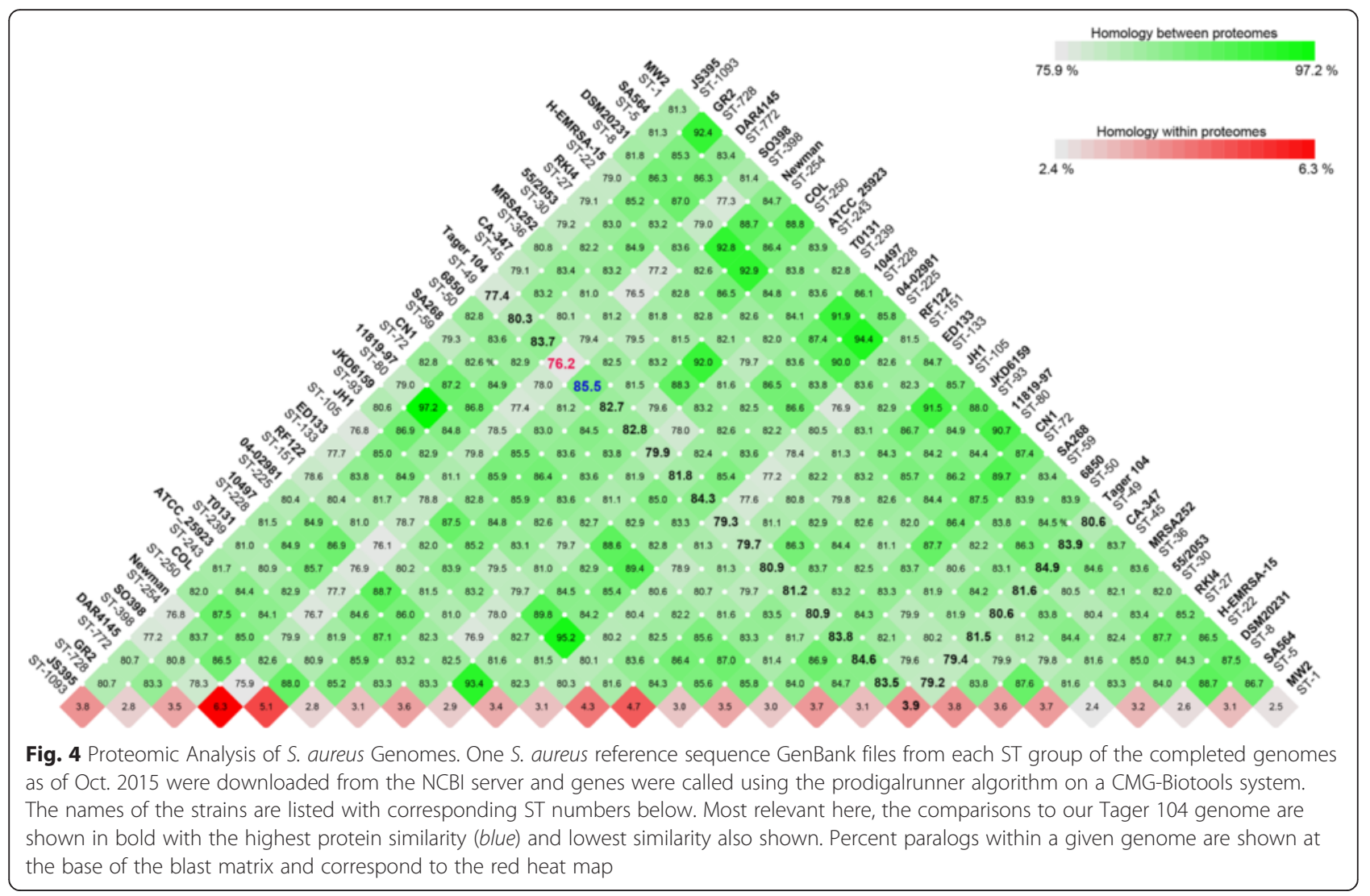

Staphylococcal inhibitor of complement (scn), which targets innate immunity by inhibiting phagocytosis by neutrophils. Tager 104 was shown to contain the hlbinterrupting phage described in the genomes of modern clinical strains, such as N315, Mu50, MW2, NCTC8325, MSSA476, MRSA252, USA300, JH1, JH9, and Newman [7], and was defined here as $\phi T G R 1$. Interestingly, Tager
104 also contains the PVL cassette (lukF-PV and lukS$P V)$, a phage-transferred pore-forming leukocyte toxin linked to necrotic infections. Tager 104 only contains one enterotoxin, determined by BLAST analysis as an exact match to the phage-transferred enterotoxin $\mathrm{P}$ [3]. PVL-positive S. aureus strains are certainly in the minority of the totality of $S$. aureus genomes sequenced to

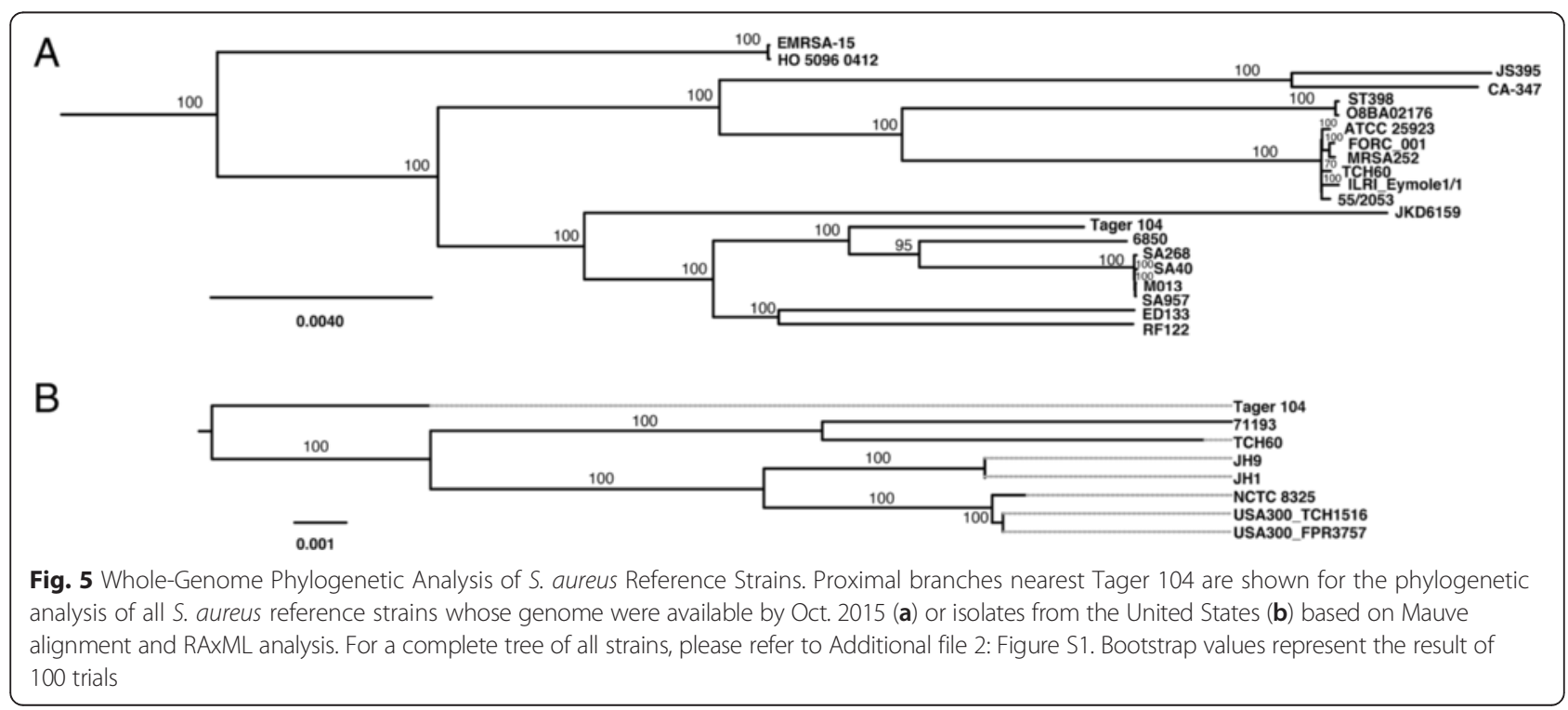




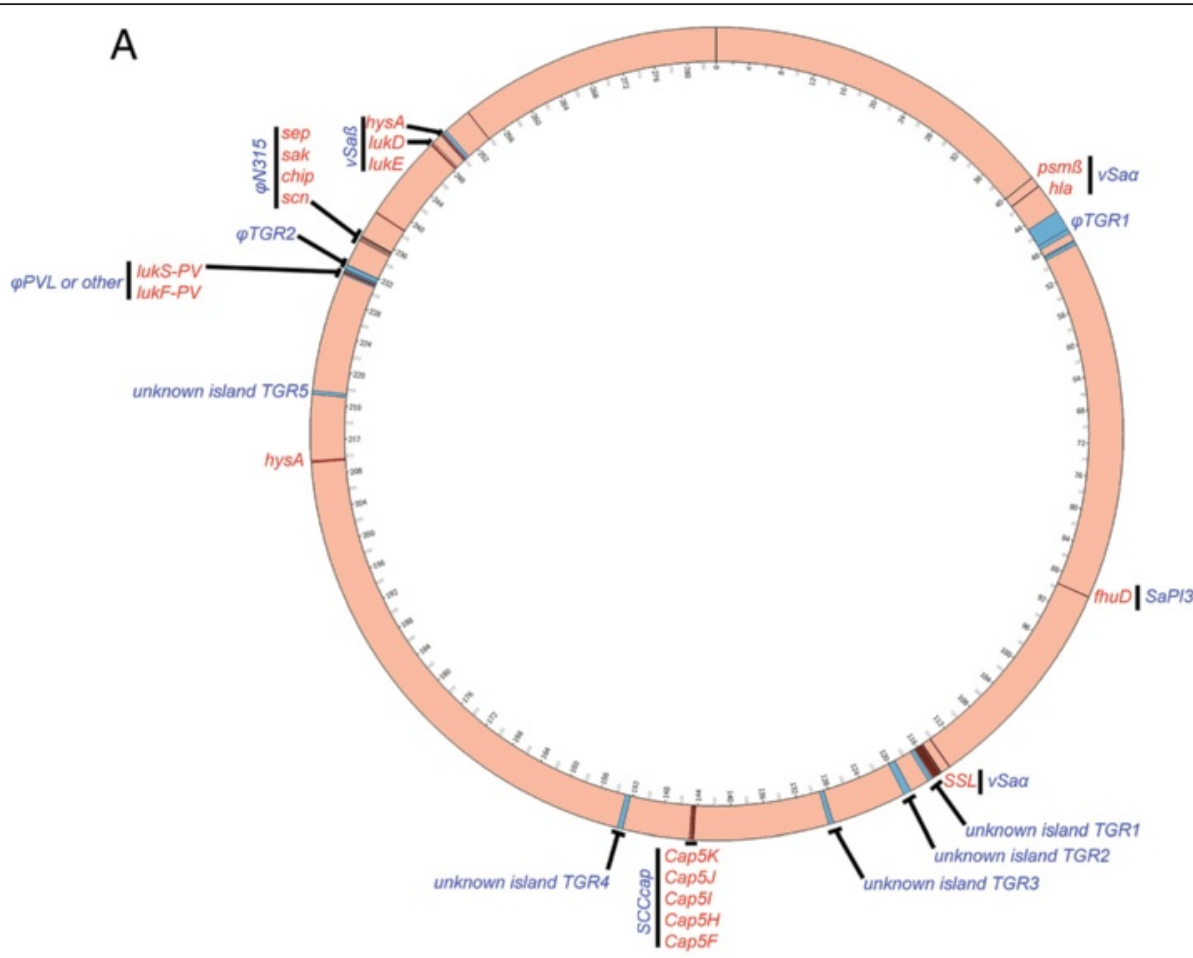

B

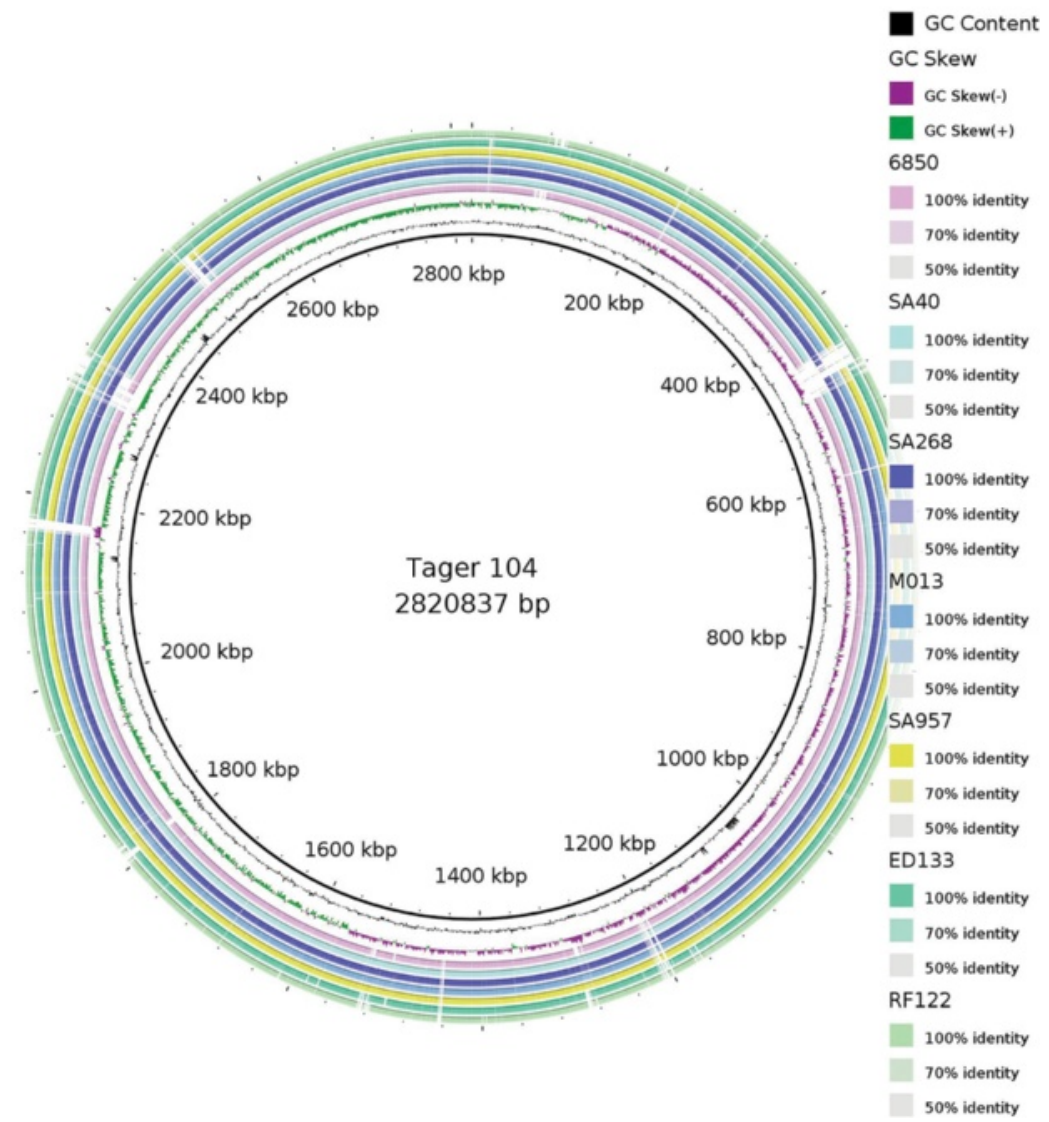

Fig. 6 (See legend on next page.) 
(See figure on previous page.)

Fig. 6 Mobile Genetic Element-Encoded Virulence in S. aureus Tager 104. a Mobile genetic element (MGE)-encoded genes relevant for virulence in S. aureus were selected based on prior knowledge of S. aureus genomics. Those elements detected by BLAST analysis in the Tager 104 genome are indicated by locus (red bands) as well as gene name (red labels), and the genomic island responsible for carrying these elements are grouped (black bar) and indicated beside the gene name (blue label). In addition, islands were detected using the IslandViewer 3 software, and are indicated by locus (blue bands) and given identifiers (blue label) based on gene identities and BLAST result. b Blast ring analysis of loci from strains in the Tager 104 clade. Tager 104 GC content and GC skew are represented on the innermost ring, with percent identity reported for genomes in the Tager 104 clade reported on the outermost rings as indicated

date, but some notable strains include ST1 strains (MW2 and USA400), ST8 strains (NCTC 8325, TW20, USA300, and USA500), and ST239 (TW20).

In addition, Tager 104 was determined to contain one unknown prophage and four unknown genomic islands, indicated as $\phi$ TGR2 and TGR1 through TGR4, respectively. The contents of these islands were limited to hypothetical and/or phage proteins (Additional file 3: Table S2).

\section{Tager 104 shows intermediate clinical adaptations}

One group of pathogenicity islands that are non-phage and non-SCC genomic islands are termed vSa islands, and typically contain a combination of virulence factors and either an intact or remnant recombinase [7]. Two such islands, termed $v \mathrm{SA} \alpha$ and $\nu \mathrm{SA} \beta$, were previously found to be allelic, and are therefore typed and used in conjunction with sequence type analysis for determination of strain radiation. Comparison between the profiles of the $v S A \beta$ genomic island for one community-acquired MRSA (CA-MRSA) strain (COL), three hospital-acquired MRSA (HA-MRSA) strains (MRSA252, Mu3, JH1), and two ruminant host MSSA strains (S0385, RF122), as well as Tager 104 demonstrated that $S$. aureus Tager 104 has lost the majority of the $b s a$ locus, with the exception of bsaG (Fig. 7). However, S. aureus Tager 104 has not acquired enterotoxin genes seen in other strains adapted for the hospital setting (HA-MRSA). The loss of this bsa locus, coupled with the gain of enterotoxins, has been previously hypothesized to indicate a shift from the environmental niche to one of hospital acquisition [36]. Therefore, S. aureus Tager 104 may represent an intermediate strain for the selection of this $v \mathrm{SA} \beta$ profile in the hospital setting.

\section{S. aureus Tager 104 antibiotic resistance profile}

To determine the potential of Tager 104 for the future study of acquired antibiotic resistance, known MGEtransferred resistance cassettes [3] were searched against the completed genome using BLAST. No resistance elements were detected (data not shown).

Minimum inhibitory concentrations (MIC) for S. aureus Tager 104 were determined by challenging with Etest strips for linezolid, vancomycin, erythromycin, and oxacillin. MICs for antibiotics were determined by
E-test strip analysis and S. aureus Tager 104 demonstrated resistance to $\mathrm{LZ}$ at $1 \mu \mathrm{g} / \mathrm{mL}, \mathrm{VA}$ at $2-3 \mu \mathrm{g} / \mathrm{mL}$, $\mathrm{ER}$ at $0.064 \mu \mathrm{g} / \mathrm{mL}$, and $\mathrm{OX}$ at $0.019 \mu \mathrm{g} / \mathrm{mL}$ (Fig. 8). The vancomycin-intermediate susceptibility phenotype of S. aureus Tager 104 was confirmed by a solutionbased test in a 96-well format; results confirm the MIC to be about $3 \mu \mathrm{g} / \mathrm{mL}$. S. aureus Tager 104 colonies on brain-heart agar plates are well-formed with the absence of satellite projections that would denote the presence of small colony variants and an overall white color, which is unlike other vancomycinintermediate strains described previously [37].

\section{Discussion}

S. aureus infections range in presentation from localized skin infections to more life-threatening osteomyelitis, endocarditis, and sepsis. With the advent of nextgeneration sequencing, analysis is now possible for individual genomes across the spectrum of infections. These data revealed that certain S. aureus strains exhibit considerable changes in their gene make-up, thus reflecting selective pressure to infect a certain host preferentially [38]. The acquisition of MGE and smaller changes in genes known as nucleotide polymorphisms together confer the apparent selective advantages needed for these strains to survive in a given host. The capacity for lateral gene transfer in $S$. aureus strains fuels this potential for rapid adaptation. For example, S. aureus Newman, isolated in 1952, has been shown to contain four prophages ( $\phi N M 1-\phi N M 4)$ and one additional vSa island, vSa4 [7]. The lack of these prophages was associated with decreased abscess formation in organs. However, a strain such as MW2 lack these prophages, but was isolated from a patient with abscesses of the brain, heart, liver, and kidneys, leading to death [4, 39]. Therefore, the required machinery for a particular infection is highly model-specific, and we suggest that reference strains should be limited in scope to those which suffice for a particular infection model.

Our previous results [30, 31, 40] suggested that $S$. aureus Tager 104 is a potent strain for the formation of bacterial endocarditis, a serious complication of bacteremia. This may not be surprising considering Tager 104 was originally isolated and maintained for decades, due in no small part for its potent ability to form stable clots in 


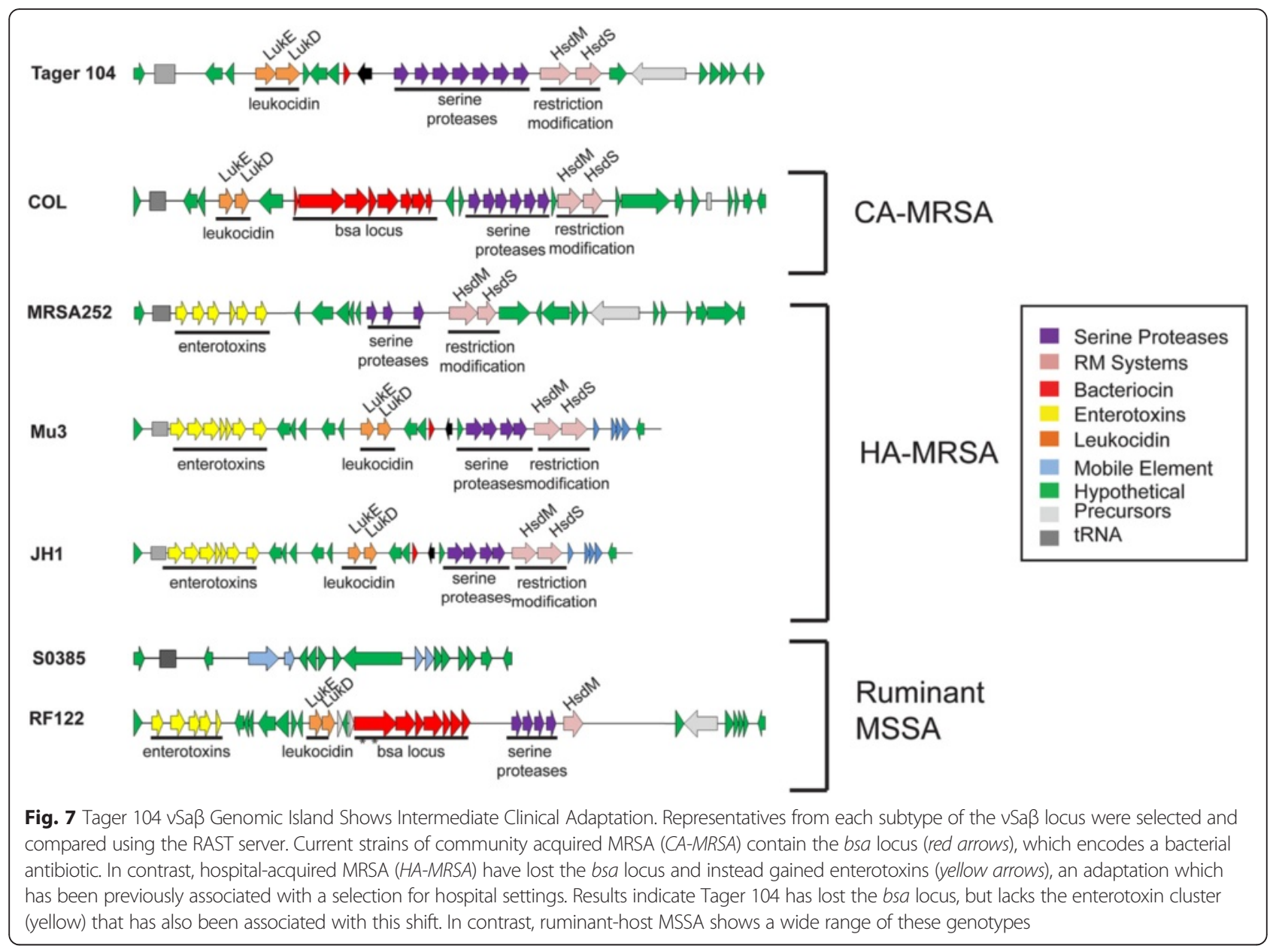

human, rabbit, and sheep blood [41]. This clotting phenotype has been attributed to the coordinated effort of two non-proteolytic activators of prothrombin, namely staphylocoagulase [16] and von Willebrand factor binding protein $[42,43]$. Both of these factors activate prothrombin by forming a reversible complex that cleaves their substrate fibrinogen to provide the fibrin barrier, similar to those seen here in the focal abscesses in multiple organs, including the kidney and spleen, that were formed by simple induction of the infection by diffuse bacteremia (Fig. 1). Because of these observations, and the early date of its isolation, we sought to outline Tager 104 as a reference strain for the systemic infection niche. We therefore determined the phylogenetic relationship of Tager 104 for future evolutionary and correlation studies compared to other $S$. aureus strains.

Our goal in genome sequencing was to find the simplest and most inexpensive combination of technologies to complete the Tager 104 genome so that it may be used as a reference for future $S$. aureus genomic comparisons. We began with Illumina MiSeq $2 \times 150$ pairedend libraries, constructed using a Nextera kit. To close the genome, literature suggested that the use of PacBio
RS technology would enable bridging across complex regions using longer reads [44]. PacBio reads were corrected using Illumina MiSeq paired-end reads, and scaffolded to create the final genomic construction. The result from this "hybrid assembly" was twelve scaffolds, which have been previously described [19]. However, no combination of algorithms or manual assembly were able to close the genome. New advancements in PacBio construction, ushered by the release of the newer HGAP assembly algorithm, led us instead to reconstruct the genome using raw PacBio data alone, producing 8 scaffolds of varied sizes, which similarly did not result in a complete genome and bridging these contigs manually proved problematic. We therefore decided to approach the closing of the genome using greater distances between the paired reads to provide additional information to the construction algorithms. Two Lucigen $20 \mathrm{~kb}$ mate-pair libraries were sufficient to close the Tager 104 genome from this HGAP-assembled data. Furthermore, Illumina $2 \times 250$ paired-end libraries were constructed, sequenced, and assembled de novo. Upon addition of the Lucigen mate-pair libraries, the final genome was produced. Therefore, the aforementioned Lucigen mate-pair 

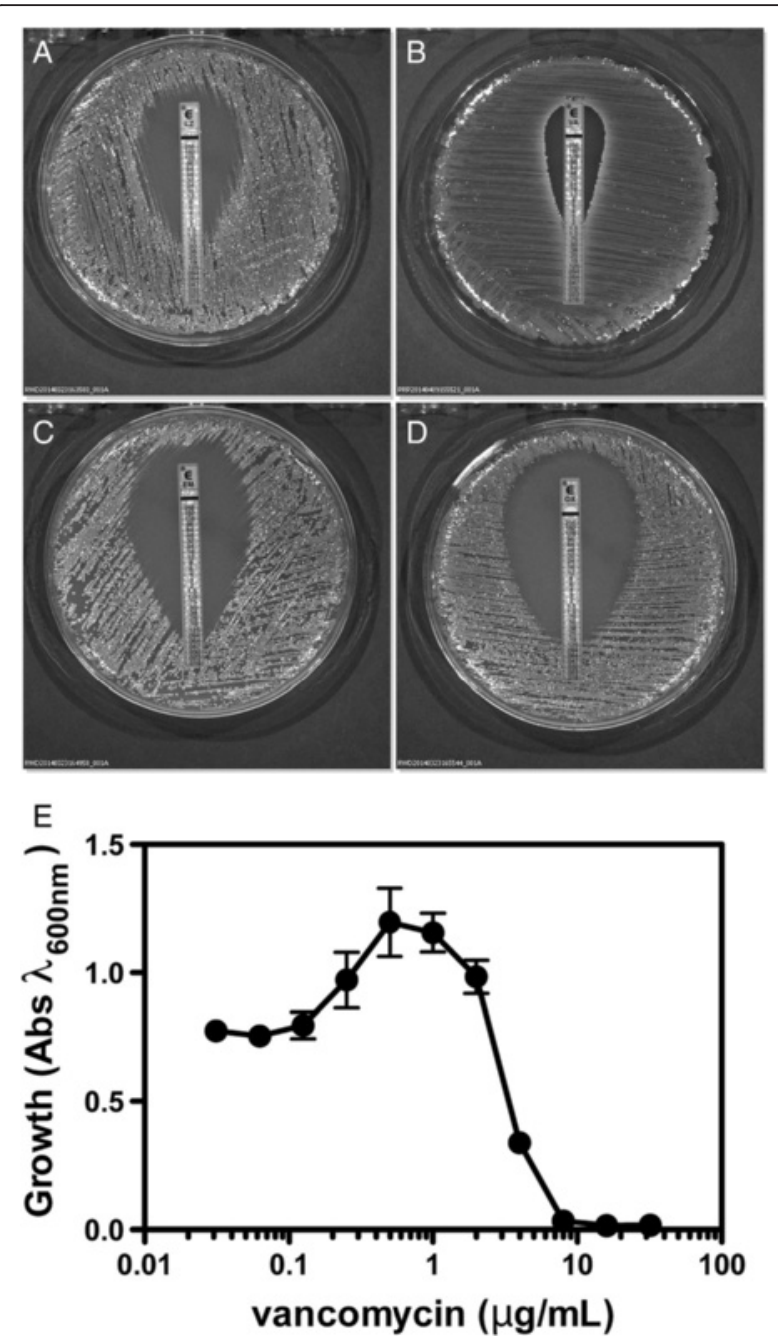

Fig. 8 S. aureus Tager 104 Susceptibility to Antibiotic Therapy Demonstrates Predation of Resistance Development. a-d Antibiotic susceptibility testing on sheep blood agar (SBA) plates demonstrated S. aureus Tager 104 is susceptible to linezolid (a), vancomycin (b), erythromycin (c), and oxacillin (d) when challenged with E-test strips. Minimum Inhibitory concentrations shown are: linezolid, 1 $\mu \mathrm{g} / \mathrm{mL}$; vancomycin, 2-3 $\mu \mathrm{g} / \mathrm{mL}$; erythromycin, $0.064 \mu \mathrm{g} / \mathrm{mL}$; and oxacillin, $0.019 \mu \mathrm{g} / \mathrm{mL}$. e The effects of vancomycin concentrations on Tager 104 growth were confirmed in solution in 96-well plates and indicate a vancomycin intermediate susceptibility phenotype. This experiment is a combination of 8 replicates and was performed as described in "Methods"

library could be used to independently assemble the Tager 104 MiSeq $2 \times 250$ bp reads. This provides a proof of principle to guide a streamlined protocol for assembly.

To interpret this $S$. aureus Tager 104 genomic dataset in the auspices of the systemic niche, it will need to have the following characteristics: (1) be a clinical sample, (2) be an early branching member of the $S$. aureus phylogenetic tree, and (3) be limited in MGE content to those which are necessary for systemic survival (i.e., immune system evasion). Based on the results of these analyses, Tager 104 has been found to have all of these characteristics. Tager 104 has been maintained from primary freezer stocks since its isolation in the late 1940's. Tager 104 shows ancestral phylogeny similar to other methicillin-resistant and methicillin-susceptible strains that have been characterized in the literature [45]. This clade is made up of two systemic isolates (6850 and SA957), one wound isolate (M013), one nasopharygenal isolate (SA40), and two ruminant isolates (ED133 and RF122). Interestingly, SA957 occurs later in the lineage than SA40 and has been associated with more severe septic infections and higher mortality rates [46]. This differentiation was hypothesized to be due to the loss of the $\beta$-hemolysin-interrupting phage.

Tager 104 shows a limited set of MGE content primarily focused on the evasion of host innate immunity. Tager 104 was shown to contain the $h l b$-interrupting phage described above, labeled as $\phi T$ TR1, as well as two additional phages. One of these phages contained PVL, indicating the earliest acquisition of this factor in the literature. The other phage was shown to carry no additional genes aside from those necessary for phage construction and replication (Additional file 3: Table S2). For comparison, prophage $\phi$ TGR1 and $\phi$ TGR2 were compared proteomically using BLAST matrix analysis with complete phage genomes downloaded from the GenBank server, and showed low homology to three phage genomes (Additional file 4: Figure S2). In addition, Tager 104 was shown to contain the $v \mathrm{Sa} \alpha$ and $v \mathrm{Sa} \beta$ islands, found in all $S$. aureus genomes [7]. Although Tager 104 was also detected to contain four additional potential genomic islands, their gene content consisted solely of hypothetical proteins. Therefore, the virulenceassociated genomic island composition of Tager 104 is limited to those which are found in all genomes ( $\mathrm{vSa}$, $v \mathrm{Sa} \beta)$, the $h l b$-interrupting phage found in most all human or animal pathogenic S. aureus ( $\phi$ TGR1), and the PVLcarrying phage strains.

The S. aureus Tager 104 genome also indicates that this strain pre-dates the acquisition of the SCCmec cassette and may serve to bridge certain clinical MRSA and MSSA strains (Fig. 5 and Additional file 2: Figure S1). Supportive to this hypothesis is the recent observation of an ST49-t208 strain that has become methicillinresistant [47], potentially through the acquisition of the mecALGA251 cassette [48]. These results indicate that Tager 104 represents an intermediate departure in the evolution of multi-drug resistant $S$. aureus. Antibiotic susceptibility of $S$. aureus Tager 104 presented here using clinically approved E-test analysis corroborates genomic data (Fig. 8) and indicates that Tager 104 displays a borderline vancomycin intermediate $S$. aureus phenotype (Fig. 8b \& e). In fact, current vancomycin guidelines would prohibit use of vancomycin in patients 
where the isolated strain MIC for vancomycin is equal to or over $2 \mu \mathrm{g} / \mathrm{mL}$ making any potential enhancement to vancomycin resistance or small colony variant formation by Tager 104 could be of significant importance to the understanding of acquired S. aureus resistance.

Interestingly, Tager 104 also shows an intermediate adaptation to the nosocomial environment. Tager 104 proteome analysis (Fig. 4) showed an equivalent homology to both community acquired strain SA268 $(84.6 \%)$ and nosocomial strain 04-02981 (84.3\%). Therefore, we investigated further the $v \mathrm{Sa} \beta$ genomic island, which has been previously implicated in the shift to hospital settings. Our analysis (Fig. 7) revealed that Tager 104 has lost the genes necessary for competition for resources in the environment, but had yet to gain those necessary for spread in a clinical setting at its time of isolation.

It has been recently suggested that the acquisition of these MGEs may be driven by "glycocodes" encoded by the teichoic acid structure of $S$. aureus, as these structures are recognized by bacteriophages during transduction events [49]. With this in mind, we investigated the locus of teichoic acid biosynthesis in S. aureus Tager 104, COL (as a representative of early MRSA strains) and JH1 (as a representative of early VRSA strains) with the hypothesis that homology in these strains indicate a potential phage-mediated acquisition of MGEs. The results indicate that the genomic makeup of this biosynthesis cluster is identical in these three strains (Additional file 5: Figure S3). Therefore, a strain ancestral to Tager 104 acquired resistance elements from bacteriophages, leading to the evolution of more recent clinical MRSA and VRSA strains, in agreement with this phylogenetic analysis (Fig. 5 and Additional file 2: Figure S1). As expected, the PS187 genome described previously showed a very distinct profile, furthering the previous conclusion that this strain is distantly related to other known $S$. aureus lineages. Further analysis will be required to investigate the origin of resistance transfer into Tager 104 and its contribution to $S$. aureus virulence.

\section{Conclusions}

Identification of a suitable reference strain for the study of $S$. aureus pathogenicity is a crucial issue that can give both context and scope to certain single nucleotide polymorphisms present in modern S. aureus isolates. Furthermore, the study of the transfer of MGEs between closely related strains may ultimately provide insight into the underlying adaptive pressures that allow these strains to cause disease in a given host. In this article, we have demonstrated that Tager 104 displays a virulent phenotype. Phylogenetic analysis, as well as a set of MGEencoded factors limited to that which is necessary for systemic survival, suggest that Tager 104 can be used as a reference strain for future studies of $S$. aureus systemic virulence. We have also placed the Tager 104 genome in context with all 75 completed S. aureus genomes and shown similarities between Tager 104 genomic islands and other human S. aureus pathogens. Moreover, we have established a methodology for the closing of complex genomic repeat regions that may accelerate closure of other genomes in the future, thereby providing an impact to researchers outside the $S$. aureus field. Given the early original isolation of the S. aureus Tager 104, the complete characterization presented here should provide new insights into the evolution of this dangerous pathogen.

\section{Availability of supporting data}

The complete Tager 104 genome is available on GenBank as accession number CP012409. Previous scaffolds mentioned in the text may be found on GenBank as accession number AVBR00000001. Phylogenetic data is available at the following URL: http://purl.org/phylo/ treebase/phylows/study/TB2:S18752.

\section{Additional files}

\section{Additional file 1: Table S1. Bacterial Strains Used In This Study} (DOCX 212 kb)

Additional file 2: Figure S1. Complete Phylogenetic Tree of S. aureus Reference Strains. Whole-genome phylogenetic analysis of all S. aureus reference strains from Fig. 5. Related branches, such as BAA1680 and the eight ST228 strains had lower bootstrap values, as would be expected given their derivations and regional isolation, respectively. Bootstrap values represent the result of 100 trials. (PDF $180 \mathrm{~kb}$ )

Additional file 3: Table S2. Genomic Islands and Prophages in the Tager 104 Genome. (DOCX 141 kb)

Additional file 4: Figure S2. Homology of Staphylococcal Phage Proteomes to the Tager 104 Prophages. Complete genome sequences were obtained from GenBank for Staphylococcal phages and protein content was predicted using saco_convert on CMG-Biotools 4.3.24. The lists of prophage-encoded proteins for $\varphi T G R 1$ and $\varphi T G R 2$ were obtained from IslandViewer 3. These lists were submitted to BLAST matrix construction on the CMG-Biotools system. (TIF 3029 kb)

Additional file 5: Figure S3. Homologous Teichoic Acid Biosynthesis Genes in S. aureus Indicate Potential Bacteriophage Interactions. Teichoic acid structure may serve as the mechanism by which phages can recognize and transfer genomic elements. Therefore, investigation of the Tager 104 teichoic acid gene cluster revealed homology to the $\mathrm{COL}$ and JH1 strains, confirming the potential of phage-mediated acquisition of genomic elements to radiate into modern clinical strains. Putative proteins are indicated with an asterisk $\left(^{*}\right)$. The teichoic acid biosynthesis gene for PS187, which shows homology to transferase genes, is indicated with a double asterisk $\left(^{* *}\right)$. (TIF $8450 \mathrm{~kb}$ )

\section{Abbreviations}

BHI: brain-heart infusion; BLAST: Basic Local Alignment Search Tool: BRIG: BLAST Ring Image Generator; CA-MRSA: community acquired MRSA; CIPRES: Cyberinfrastructure for Phylogenetic Research; ER: erythromycin; H\&E: hematoxylin and eosin; HA-MRSA: hospital acquired MRSA; HGAP: Hierarchical Genome Assembly Process; LCB: locally collinear blocks; LZ: linezolid; MGE: mobile genetic elements; MIC: minimum inhibitory concentration; MLST: multi-locus sequence typing; MRSA: methicillinresistant S. aureus; MSSA: methicillin-sensitive S. aureus; O.D.: optical density; OCT: optimal cutting temperature; OX: oxacillin; PBS: phosphate buffered saline; PEG: protein-encoding gene; PVL: Panton-Valentine Leukocidin; RAST: Rapid Annotated using Subsystem Technology; S. 
aureus: Staphylococcus aureus; SCCmec: Staphylococcal Cassette Chromosome mec; SPAdes: St. Petersburg genome assembler; SSPACE: SSAKE-based scaffolding of Pre-Assembled Contigs after Extension; ST: sequence type; TSA: trypticase soy agar; VA: vancomycin; VRSA: vancomycin-resistant S. aureus.

\section{Competing interests}

The authors report no competing interests.

\section{Authors' contributions}

$R D, M N, P E B, M R L, D M$, and PP drafted the manuscript. RD carried out genomic construction and characterization. ADB carried out MLST analysis and aided in tree construction. PP, MRL, and MJH participated in the design of the study. SM, ML and DM constructed the Lucigen NxSeq libraries and sequenced these libraries, as well as aiding in the design of genomic construction. All authors read and approved the final manuscript.

\section{Acknowledgements}

Supported by the National Institutes of Health through grants provided by the National Heart, Lung, and Blood Institute, R00HL094533 (to P.P.), R01HL114477 (to P.P. and M.N.), R01 HL071544 (to P.E.B). Support also provided by the National Institute of Allergy and Infectious Diseases grant 2R44AI085840-02 (to D.M., P.P. and M.R.L.). The authors also wish to thank the Alabama Supercomputer Authority for access to the Alabama Supercomputer.

\section{Author details}

'Department of Drug Discovery and Development, Harrison School of Pharmacy, Auburn University, 4306 Walker Building, Auburn, AL 36849, USA.

${ }^{2}$ Department of Biological Sciences, Auburn University, 101 Rouse Life Science Building, Auburn, AL 36849, USA. 'Lucigen Corporation, 2905 Parmenter St, Middleton, WI 53562, USA. Department of Pathology, Microbiology, and Immunology, Vanderbilt University Medical Center, Nashville, TN 37232, USA. ${ }^{5}$ Center for Systems Biology, Massachusetts General Hospital and Harvard Medical School, Simches Research Building, 185 Cambridge St., Boston, MA 02114, USA.

\section{Received: 28 September 2015 Accepted: 3 February 2016} Published online: 03 March 2016

\section{References}

1. Prevention CfDCa. Antibiotic resistance threats in the United States, 2013. July 17th, 2014 ed. 2013. p. 1-114.

2. Fowler Jr VG, Miro JM, Hoen B, Cabell CH, Abrutyn E, Rubinstein E, Corey GR, Spelman D, Bradley SF, Barsic B, et al. Staphylococcus aureus endocarditis: a consequence of medical progress. JAMA. 2005;293(24):3012-21.

3. Malachowa N, DeLeo FR. Mobile genetic elements of Staphylococcus aureus. Cell Mol Life Sci. 2010;67(18):3057-71.

4. Baba T, Takeuchi F, Kuroda M, Yuzawa H, Aoki K, Oguchi A, Nagai Y, Iwama N, Asano K, Naimi T, et al. Genome and virulence determinants of high virulence community-acquired MRSA. Lancet. 2002;359(9320):1819-27.

5. Brussow H, Canchaya C, Hardt WD. Phages and the evolution of bacterial pathogens: from genomic rearrangements to lysogenic conversion. Microbiol Mol Biol Rev. 2004;68(3):560-602. table of contents.

6. Plano LR. Staphylococcus aureus exfoliative toxins: how they cause disease. J Invest Dermatol. 2004;122(5):1070-7.

7. Baba T, Bae T, Schneewind O, Takeuchi F, Hiramatsu K. Genome sequence of Staphylococcus aureus strain Newman and comparative analysis of staphylococcal genomes: polymorphism and evolution of two major pathogenicity islands. J Bacteriol. 2008;190(1):300-10.

8. Diep BA, Gill SR, Chang RF, Phan TH, Chen JH, Davidson MG, Lin F, Lin J, Carleton HA, Mongodin EF, et al. Complete genome sequence of USA300, an epidemic clone of community-acquired meticillin-resistant Staphylococcus aureus. Lancet. 2006;367(9512):731-9.

9. Kuroda M, Ohta T, Uchiyama I, Baba T, Yuzawa H, Kobayashi I, Cui L, Oguchi A, Aoki K, Nagai Y, et al. Whole genome sequencing of meticillin-resistant Staphylococcus aureus. Lancet. 2001;357(9264):1225-40.

10. Tager M, Hales HB. Quantitative coagulase and toxin production by Staphylococcus in relation to the clinical source of the organisms. Yale J Biol Med. 1947:20(1):41-9.

11. Tager M. Studies on the coagulase-reacting factor; the reaction of staphylocoagulase with the components of human plasma. Yale J Biol Med. 1948;20(4):369-80.
12. Tager M, Drummond MC. Staphylocoagulase. Ann N Y Acad Sci. 1965;128(1):92-111.

13. Tager M. Concentration, partial purification, properties, and nature of staphylocoagulase. Yale J Biol Med. 1948;20(5):487-501.

14. Tager M, Lodge AL. Influence of the physiological blood clotting process on the coagulation of blood by staphylocoagulase. J Exp Med. 1951;94(1):73-85.

15. Tager M, Lodge AL. Changes in the properties of the coagulase-reacting factor of plasma after separation from prothrombin by Seitz filtration. J Immunol. 1951;67(1):63-9.

16. Friedrich $R$, Panizzi P, Fuentes-Prior P, Richter $K$, Verhamme I, Anderson PJ, Kawabata S, Huber R, Bode W, Bock PE.. Staphylocoagulase is a prototype for the mechanism of cofactor-induced zymogen activation. Nature. 2003;425(6957):535-9.

17. Panizzi P, Friedrich R, Fuentes-Prior P, Kroh HK, Briggs J, Tans G, Bode W, Bock PE.. Novel fluorescent prothrombin analogs as probes of staphylocoagulaseprothrombin interactions. J Biol Chem. 2006:281(2):1169-78.

18. Panizzi P, Friedrich R, Fuentes-Prior P, Richter K, Bock PE, Bode W. Fibrinogen substrate recognition by staphylocoagulase. (pro)thrombin complexes. J Biol Chem. 2006;281(2):1179-87.

19. Davis R, Hossain MJ, Liles MR, Panizzi P. Complete genome sequence of Staphylococcus aureus Tager 104, a sequence type 49 ancestor. Genome Announc. 2013;1(5).

20. Chin CS, Alexander DH, Marks P, Klammer AA, Drake J, Heiner C, Clum A, Copeland A, Huddleston J, Eichler EE, et al. Nonhybrid, finished microbial genome assemblies from long-read SMRT sequencing data. Nat Methods. 2013;10(6):563-9.

21. Hunt M, Newbold C, Berriman M, Otto TD. A comprehensive evaluation of assembly scaffolding tools. Genome Biol. 2014;15(3):R42.

22. Aziz RK, Bartels D, Best AA, DeJongh M, Disz T, Edwards RA, Formsma K Gerdes S, Glass EM, Kubal M, et al. The RAST Server: rapid annotations using subsystems technology. BMC Genomics. 2008:9:75.

23. Brettin T, Davis JJ, Disz T, Edwards RA, Gerdes S, Olsen GJ, Olson R, Overbeek R, Parrello B, Pusch GD, et al. RASTtk: a modular and extensible implementation of the RAST algorithm for building custom annotation pipelines and annotating batches of genomes. Sci Rep. 2015;5:8365.

24. Overbeek R, Olson R, Pusch GD, Olsen GJ, Davis JJ, Disz T, Edwards RA, Gerdes S, Parrello B, Shukla M, et al. The SEED and the Rapid Annotation of microbial genomes using Subsystems Technology (RAST). Nucleic Acids Res. 2014:42(Database issue):D206-14.

25. Nurk S, Bankevich A, Antipov D, Gurevich AA, Korobeynikov A, Lapidus A, Prjibelski AD, Pyshkin A, Sirotkin A, Sirotkin Y, et al. Assembling single-cell genomes and mini-metagenomes from chimeric MDA products. J Comput Biol. 2013;20(10):714-37.

26. Kurtz S, Phillippy A, Delcher AL, Smoot M, Shumway M, Antonescu C, Salzberg SL. Versatile and open software for comparing large genomes. Genome Biol. 2004;5(2):R12.

27. Vesth T, Lagesen K, Acar O, Ussery D. CMG-biotools, a free workbench for basic comparative microbial genomics. PLoS One. 2013:8(4):e60120.

28. Darling AE, Mau B, Perna NT. progressiveMauve: multiple genome alignment with gene gain, loss and rearrangement. PLoS One. 2010;5(6):e11147.

29. Alikhan NF, Petty NK, Ben Zakour NL, Beatson SA. BLAST Ring Image Generator (BRIG): simple prokaryote genome comparisons. BMC Genomics. 2011;12:402.

30. Panizzi $P$, Nahrendorf $M$, Figueiredo JL, Panizzi JR, Marinelli B, Iwamoto $Y$, Keliher E, Maddur AA, Waterman P, Kroh HK, Leuschner F, Aikawa E, Swirski FK, Pittet MJ, Hackeng TM, Fuenes-Prior P, Schneedwind O, Bock PE, Weissleder R. In vitro detection of Staphylococcus aureus endocarditis by targeting pathogen-specific prothrombin activation. Nat Med. 2011;17(9):1142-6.

31. Panizzi P, Stone JR, Nahrendorf M. Molecular imaging of endocarditis. J Nucl Cardiol. 2014:21:486-95

32. Feil EJ, Li BC, Aanensen DM, Hanage WP, Spratt BG. eBURST: inferring patterns of evolutionary descent among clusters of related bacterial genotypes from multilocus sequence typing data. J Bacteriol. 2004;186(5):1518-30.

33. Schijffelen MJ, Boel CH, van Strijp JA, Fluit AC. Whole genome analysis of a livestock-associated methicillin-resistant Staphylococcus aureus ST398 isolate from a case of human endocarditis. BMC Genomics. 2010; $11: 376$

34. Fraunholz M, Bernhardt J, Schuldes J, Daniel R, Hecker M, Sinha B. Complete Genome Sequence of Staphylococcus aureus 6850, a Highly Cytotoxic and Clinically Virulent Methicillin-Sensitive Strain with Distant Relatedness to Prototype Strains. Genome Announc 2013, 1(5). 
35. Powers ME, Bubeck Wardenburg J. Igniting the fire: Staphylococcus aureus virulence factors in the pathogenesis of sepsis. PLoS Pathog. 2014;10(2):e1003871.

36. Kos VN, Desjardins CA, Griggs A, Cerqueira G, Van Tonder A, Holden MT, Godfrey P, Palmer KL, Bodi K, Mongodin EF, et al. Comparative genomics of vancomycin-resistant Staphylococcus aureus strains and their positions within the clade most commonly associated with Methicillin-resistant $S$. aureus hospital-acquired infection in the United States. mBio. 2012;3(3): e00112-12. doi:10.1128/mBio.00112-12.

37. Howden BP, Davies JK, Johnson PD, Stinear TP, Grayson ML. Reduced vancomycin susceptibility in Staphylococcus aureus, including vancomycinintermediate and heterogeneous vancomycin-intermediate strains: resistance mechanisms, laboratory detection, and clinical implications. Clin Microbiol Rev. 2010;23(1):99-139.

38. Herron-Olson L, Fitzgerald JR, Musser JM, Kapur V. Molecular correlates of host specialization in Staphylococcus aureus. PLoS One. 2007;2(10):e1120.

39. Centers for Disease C, Prevention. Four pediatric deaths from community-acquired methicillin-resistant Staphylococcus aureus Minnesota and North Dakota, 1997-1999. MMWR Morb Mortal Wkly Rep. 1999:48(32):707-10.

40. Eggleston $\mathrm{H}$, Panizzi P. Molecular imaging of bacterial infections in vivo: the discrimination between infection and inflammation. Informatics. 2014;1:72-99

41. Woods CW, Cheng AC, Fowler Jr VG, Moorefield M, Frederick J, Sakoulas G, Meka VG, Tenover FC, Zwadyk P, Wilson KH. Endocarditis caused by Staphylococcus aureus with reduced susceptibility to vancomycin. Clin Infect Dis. 2004;38(8):1188-91.

42. Panizzi P, Friedrich R, Fuentes-Prior P, Bode W, Bock PE. The staphylocoagulase family of zymogen activator and adhesion proteins. Cell Mol Life Sci. 2004;61(22):2793-8.

43. Kroh HK, Panizzi P, Bock PE. Von Willebrand factor-binding protein is a hysteretic conformational activator of prothrombin. Proc Natl Acad Sci U S A. 2009:106(19):7786-91.

44. Koren S, Schatz MC, Walenz BP, Martin J, Howard JT, Ganapathy G, Wang Z, Rasko DA, McCombie WR, Jarvis ED, et al. Hybrid error correction and de novo assembly of single-molecule sequencing reads. Nat Biotechnol. 2012;30(7):693-700.

45. Benson MA, Ohneck EA, Ryan C, Alonzo 3rd F, Smith H, Narechania A, Kolokotronis SO, Satola SW, Uhlemann AC, Sebra R, et al. Evolution of hypervirulence by a MRSA clone through acquisition of a transposable element. Mol Microbiol. 2014;93(4):664-81.

46. Chen CJ, Unger C, Hoffmann W, Lindsay JA, Huang YC, Gotz F. Characterization and comparison of 2 distinct epidemic communityassociated methicillin-resistant Staphylococcus aureus clones of ST59 lineage. PLoS One. 2013;8(9):e63210.

47. Overesch G, Buttner S, Rossano A, Perreten V. The increase of methicillinresistant Staphylococcus aureus (MRSA) and the presence of an unusual sequence type ST49 in slaughter pigs in Switzerland. BMC Vet Res. 2011;7:30,

48. Paterson GK, Larsen AR, Robb A, Edwards GE, Pennycott TW, Foster G, Mot D, Hermans K, Baert K, Peacock SJ, et al. The newly described mecA homologue, mecALGA251, is present in methicillin-resistant Staphylococcus aureus isolates from a diverse range of host species. J Antimicrob Chemother. 2012;67(12):2809-13.

49. Winstel V, Liang C, Sanchez-Carballo P, Steglich M, Munar M, Broker BM, Penades JR, Nubel U, Holst O, Dandekar T, et al. Wall teichoic acid structure governs horizontal gene transfer between major bacterial pathogens. Nat Commun. 2013:4:2345

\section{Submit your next manuscript to BioMed Central and we will help you at every step:}

- We accept pre-submission inquiries

- Our selector tool helps you to find the most relevant journal

- We provide round the clock customer support

- Convenient online submission

- Thorough peer review

- Inclusion in PubMed and all major indexing services

- Maximum visibility for your research

Submit your manuscript at www.biomedcentral.com/submit 